Manuelle Medizin 2015 $53: 480$

DOI 10.1007/s00337-015-0065-8

(C) Springer-Verlag

Berlin Heidelberg 2015

\title{
$\nabla_{\Delta}^{\nabla} \Delta_{\Delta} \Delta^{\top} \Delta \nabla$ \\ MANUELLEMEDIZIN \\ SAMM
}

\section{Schweizerische Ärztegesellschaft für Manuelle Medizin SAMM}

Weiterbildung in Manueller Medizin

Anmeldung und Auskünfte:

Sekretariat SAMM

Kolumbanstrasse 2

9008 St.Gallen
Tel. 0712465181

Fax. 0712465101

info@samm.ch

www.samm.ch

\section{Kursdaten bei Ausbildungsbeginn im Jahr 2015}

Modul 5 HWS/zervikothorakaler Übergang, 13.-16.01.2016 Ellbogen/Hand

Erweiterung Therapie

mit Schwerpunkt MMI

Modul 6

Zervikothorakaler und thora-

kolumbaler Übergang, Fuss

Erweiterung MMI/MOI/NMI,

Triggerpunkt-Therapie

Modul 7

Vertiefung,

regionaler Beschwerdebilder

Gesamtrepetition anhand regionaler

Beschwerdebilder

Modul 8

Klinische Integration

21.-24.09.2016 oder mit Patienten-Präsentation,

28.09.-01.10.2016

Gesamtrepetition, Prüfungsvorbereitung

Weiterbildung bei Ausbildungsbeginn im Jahr 2016

Modul 1

HWS, Schultergürtel

06. -09.01 .2016$

Diagnostik \& Einführung Therapie

MOI/NMI, Neurodynamik,

Muskulatur, Schulter

Modul 2

LWS, Beckenring

20.-23.03.2016

Diagnostik \& Einführung Therapie

MOI/NMI, Neurodynamik, Muskulatur,

Hüfte, Knie

Modul 3

BWS, Rippen

15. -18.06 .2016$

Diagnostik \& Therapie MOI/NMI,

Rumpfstabilisierung

Modul 4 LWS, Beckenring, BWS-Rippen,

erste schriftl. Teilprüfung (1. Tag),

Erweiterung Therapie

mit Schwerpunkt MMI

14. -17.09 .2016$

* Der reguläre Kursstart findet im Januar statt. Alternativ und besonders für angehende Fachärzte R/PMR besteht die Möglichkeit, zuerst die ganze muskulo-skelettale Diagnostik und Einführung in die Manuelle Therapie zu absolvieren (Modul 1 bis 3 ) sowie bei Bedarf zu einem späteren Zeitpunkt mit der Ausbildung fortzufahren.

\section{Kongresse für Manuelle Medizin}

57. Jahreskongress SAMM 2016

24.-26. November 2016, Interlaken
Update regional $1 \mathrm{Tag}-2016$

Sa. 02.04. Thorakozervikaler und thorakolumbaler

9:00-16:00 Übergang erfolgreich behandeln

Berner Fachhochschule, M. Lauper,

8 Credits, CHF 200.-

Mi. 24. 08.** „Von Kopf bis Fuss“

9:30-17:00 Klinik St.Katharinental, Diessenhofen*, Th. Tritschler, 8 Credits, CHF 200.-

\section{Sa. 01.10. LWS}

9:30-16:30 Clinique Suva Care, Sion, M.H. Gauchat, 8 Credits, CHF 200.-

Update regional $1 / 2$ Tag - 2016

Do. 07.04. ${ }^{* *}$ HSA

17:00-20:00 Bethesda Spital, Basel ${ }^{\star}$, M. Baumann, B. Zeybeker,

4 Credits, CHF 100.-

Mi. 20.04. HWS

18:00-21:00 Schulthess Klinik, Zürich, M. Bitterli, G. Hämmerle, 4 Credits, CHF 100.-

Do. 21.04. ${ }^{* *}$ Becken und ISG

17:00-20:00 Clinique Suva Care, Sion,

D. Margni, F. Vanini, G. Maffei, 4 Credits gesponsert

Sa. 07.05. HSA und obere Extremitäten

13:00-16:00 Praxis Dr. Sager, Winterthur, M. Sager,

4 Credits, CHF 100.-

Mi. 31.08. Cervicothorakaler Uebergang

18:00-21:00 Schulthess Klinik, Zürich, M. Bitterli, G. Hämmerle, 4 Credits, CHF 100.-

Do. 15.09. ${ }^{* *}$ LWS

15:30-18:30 Clinica Novaggio TI, D. Margni, F. Vanini, G. Maffei,

4 Credits, gesponsert

Sa. 24.09. LBB und untere Extremitäten

13:00-16:00 Praxis Dr. Sager, Winterthur, M. Sager,

4 Credits, CHF 100.-

Do. 29.09.** LBB

17:00-20:00 Bethesda Spital, Basel* ${ }^{\star}$, St. Bürgin, B. Zeybeker, 4 Credits, gesponsert

Abmeldebedingungen Update: 10 Tage vor Kursbeginn $=100 \%$ der Kurskosten

** mit Teilnahme von SAMT-Physiotherapeuten

\section{Fortbildungsmodule 2016}

(Detaillierte Informationen zu FM1/FM2 finden Sie auf www.samm.ch)

08.-09.04.

FM1: MOI/NMI

Centre Löwenberg, Murten, tbd, 16 Credits,

Kosten $^{\star *}$ ohne Unterkunft: CHF 800.-

Kosten $^{\star *}$ inkl. Unterkunft + VP: CHF 904.-

08.-09.04.

\section{FM2: MOI/NMI}

Centre Löwenberg, Murten, tbd, 16 Credits,

Kosten $^{\star *}$ ohne Unterkunft: CHF 800.-

Kosten $^{\star *}$ inkl. Unterkunft + VP: CHF 904.-

Abmeldebedingungen FM1/FM2: 30 Tage vor Kursbeginn $=50 \%$ der Kurskosten,

12 Tage vorher $=80 \%$, 6 Tage vorher $=100 \%$

** Nicht-Mitglieder bezahlen einen Zuschlag von CHF 50.-/Tag 